\title{
CRISPR-Cas9 disruption of PD-1 enhances activity of universal EGFRvIlI CAR T cells in a preclinical model of human glioblastoma
}

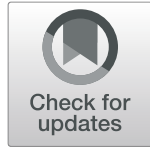

\author{
Bryan D. Choi ${ }^{1,2}$, Xiaoling Yu', Ana P. Castano ${ }^{1}$, Henia Darr ${ }^{3}$, Daniel B. Henderson ${ }^{3}$, Amanda A. Bouffard ${ }^{1}$, \\ Rebecca C. Larson", Irene Scarfò ${ }^{1}$, Stefanie R. Bailey ${ }^{1}$, Genevieve M. Gerhard ${ }^{1}$, Matthew J. Frigault ${ }^{1,4}$, Mark B. Leick', \\ Andrea Schmidts ${ }^{1}$, Jason G. Sagert ${ }^{3}$, William T. Curry ${ }^{2}$, Bob S. Carter ${ }^{2}$ and Marcela V. Maus ${ }^{1,4^{*}}$ (D)
}

\begin{abstract}
Despite remarkable success in the treatment of hematological malignancies, CAR T-cell therapies for solid tumors have floundered, in large part due to local immune suppression and the effects of prolonged stimulation leading to T-cell dysfunction and exhaustion. One mechanism by which gliomas and other cancers can hamper CAR T cells is through surface expression of inhibitory ligands such as programmed cell death ligand 1 (PD-L1). Using the CRIPSRCas9 system, we created universal CAR T cells resistant to PD-1 inhibition through multiplexed gene disruption of endogenous T-cell receptor (TRAC), beta-2 microglobulin (B2M) and PD-1 (PDCD1). Triple gene-edited CAR T cells demonstrated enhanced activity in preclinical glioma models. Prolonged survival in mice bearing intracranial tumors was achieved after intracerebral, but not intravenous administration. CRISPR-Cas9 gene-editing not only provides a potential source of allogeneic, universal donor cells, but also enables simultaneous disruption of checkpoint signaling that otherwise impedes maximal antitumor functionality.
\end{abstract}

Keywords: CRISPR-Cas systems, Receptors, chimeric antigen, EGFRvIll, Glioblastoma

Glioblastoma (GBM) is the most common primary malignant brain tumor and it is also the most aggressive [1]. Despite standard-of-care multimodal therapy, over $70 \%$ of patients with GBM die within 2 years of diagnosis [2]. T-cell immune therapy represents an emerging alternative to conventional treatment, and has been shown to successfully treat solid tumors in the brain, even in the setting of bulky and invasive disease [3]. One of the most promising $\mathrm{T}$-cell platforms is the chimeric antigen receptor (CAR), which has revolutionized the treatment and management of hematological malignancies with first-in-class approval by the Food and Drug Administration in 2017 [4]. However, the efficacy of CAR $\mathrm{T}$ cells has not been successfully translated to the setting of GBM to date [5]. One explanation for this

\footnotetext{
* Correspondence: mvmaus@mgh.harvard.edu

${ }^{1}$ Cellular Immunotherapy Program, Cancer Center, Massachusetts General Hospital and Harvard Medical School, 149 13th Street, Room 3.216, Charlestown, Boston, Massachusetts 02129, USA

${ }^{4}$ Department of Medicine, Massachusetts General Hospital and Harvard Medical School, Boston, Massachusetts, USA

Full list of author information is available at the end of the article
}

includes the profound local and systemic immune suppression observed in patients with GBM. Moreover, autologous CAR $\mathrm{T}$-cell production remains costly and time-consuming, and it can be challenging to control disease progression in GBM patients while their $\mathrm{T}$ cells are being manufactured. To this end, off-the-shelf CAR $\mathrm{T}$ cells that are resistant to local immune suppression could have meaningful benefit.

In our clinical study of intravenous CAR T cells targeting a tumor-specific mutation of the epidermal growth factor receptor (EGFRvIII) in patients with GBM, we observed that EGFRvIII CAR T cells localized to intracerebral tumors and led to successful reduction of EGFRvIIIexpressing cancer cells [6]. However, this was also associated with concomitant upregulation of programmed cell death ligand 1 (PD-L1) expression within treated gliomas, ultimately contributing to immune suppression, CAR T-cell dysfunction and subsequent disease progression. In addition, four out of 17 subjects did not receive CAR $\mathrm{T}$ cells in the trial due to rapid disease progression, highlighting the potential benefit of "off-the-shelf," 
ready-to-use products that do not otherwise require custom generation [6].

CRIPSR-Cas9 technology has emerged as a simple and efficient method of gene-editing CARs with the potential to address these barriers to therapy. This includes the design of universal CAR $\mathrm{T}$ cells with reduced potential for both initiating graft-versus-host disease (GVHD) and eliciting donor T-cell rejection, through targeted disruption of the endogenous $\mathrm{T}$-cell receptor $(T R A C)$ and beta-2 microglobulin $(B 2 M)$, respectively $[7,8]$. The use of CRISPR-Cas9 also affords the opportunity to modify the expression of other relevant genes involved in suppressing $\mathrm{T}$-cell function in the microenvironment of GBM tumors.

In the current study, we applied CRISPR-Cas9 to generate an allogeneic EGFRvIII CAR T-cell product deficient in TCR and B2M. We also simultaneously disrupted endogenous PD-1 (PDCD1), thereby averting the potential effects of post-treatment PD-L1 upregulation in gliomas that was observed in the clinical trial. Here, we demonstrate that multiplexed geneediting for TRAC, B2M and PDCD1 can be performed efficiently in primary human $\mathrm{T}$ cells prior to CAR transduction. In addition, we observed that the antitumor efficacy of gene-edited EGFRvIII CAR T cells is enhanced by targeted disruption of PD-1 in preclinical models of GBM.

\section{Results}

Multiplexed gene-editing of EGFRvIII CAR T cells

In the current study, we employed the EGFRvIII CAR Tcell construct based on a second-generation backbone containing 4-1BB and $\mathrm{CD} 3 \zeta$ intracellular signaling domains, but this time cloned into an AAV6 vector backbone instead of a lentiviral vector (Fig. 1a), the former allowing for integration of the CAR sequence into a specific locus rather than relying on random genomic integration. Briefly, the strategy for multiplexed gene-editing consists of in vitro stimulation of primary human $\mathrm{T}$ cells, followed by electroporation with respective Cas9 ribonucleoproteins (RNPs) and subsequent adeno-associated virus (AAV)-mediated transduction of the CAR (Fig. 1b). CRISPR-Cas9 gene-editing using RNP electroporation for TRAC and B2M genetic loci was efficient, yielding populations of greater than $80 \%$ double knock-out surface expression by flow cytometry (Fig. 1c,d). In a separate experimental group, RNP electroporation was multiplexed to generate $\mathrm{T}$ cells also edited for $P D C D 1$, in addition to TRAC and B2M. This was followed by AAV6 transduction, which resulted in CAR T cells with either endogenous or deleted PD-1 (i.e., CART-EGFRvIII and CARTEGFRvIII $\triangle$ PD-1) (Fig. 1e). Following stimulation with EGFRvIII-expressing glioma, we demonstrated that both control (i.e., T cells edited for TRAC and $B 2 M$, without CAR) and CART-EGFRvIII cells (i.e., $\mathrm{T}$ cells edited for

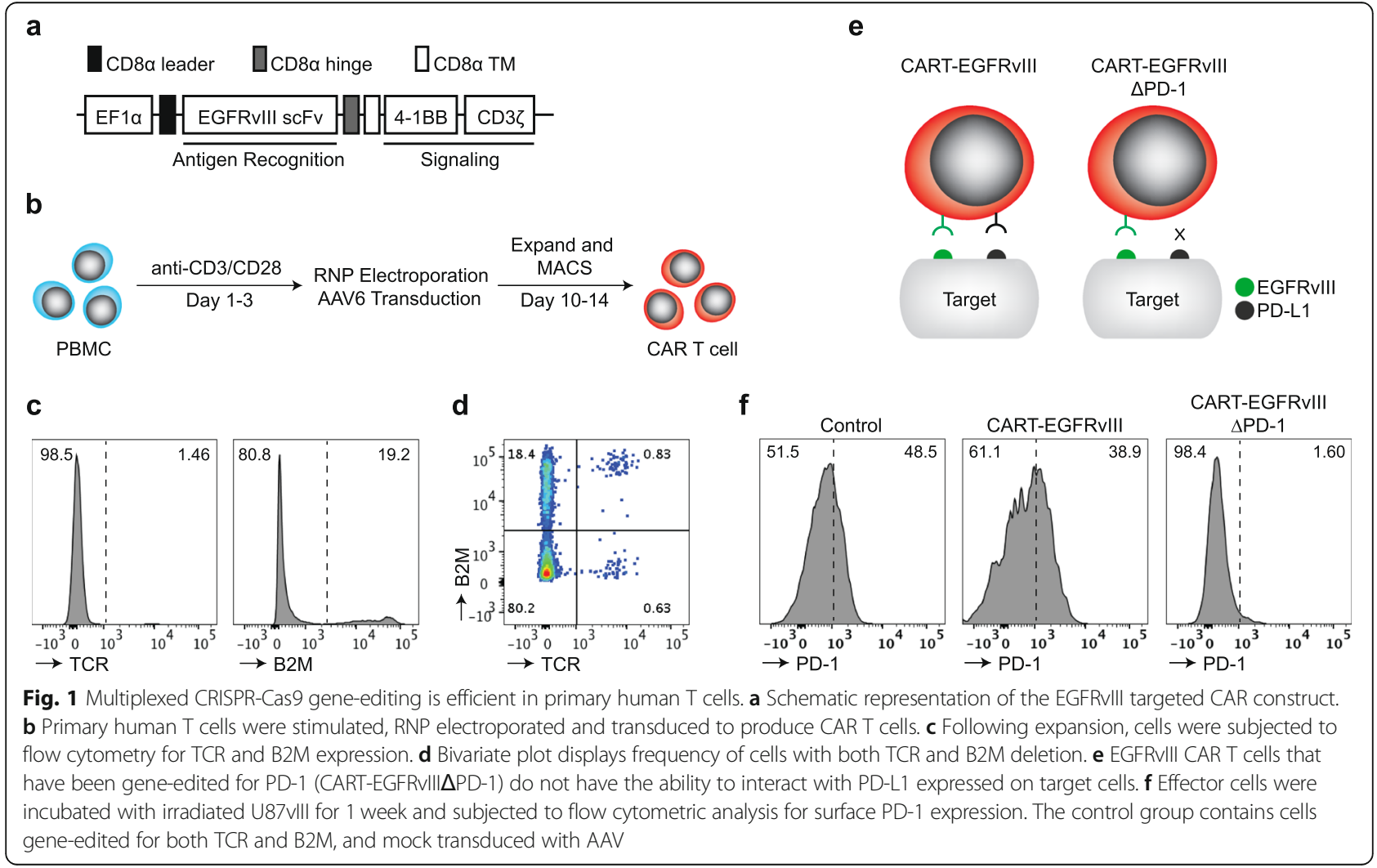


TRAC and B2M, with CAR) were positive for surface PD1 by flow cytometry. By contrast, PD-1 was not detected on the surface of CART-EGFRvIII $\triangle \mathrm{PD}-1$ cells, confirming effective knock-out at the level of surface protein expression in the entire population (Fig. 1f).

\section{CAR T-cell differentiation following CRISPR-Cas9 gene- editing}

We next sought to assess the levels of PD-L1 expression on commonly used brain tumor cell lines. Importantly, PD-L1 has been shown to be frequently found on the surface of GBMs [9] and upregulated in patients treated with EGFRvIII CAR T cells [6]. To demonstrate proof-of-concept, we selected a wellcharacterized EGFRvIII-positive glioma line, U87vIII, as a canonical target cell for our study. Compared to its parental line, U87, and another commonly used glioma cell line, U251, we demonstrated that U87vIII naturally expresses PD-L1; however, this appeared to be decreased relative to U87 and U251 by flow cytometric analysis (Additional file 1: Figure S1).

We then proceeded to assess the impact of CRISPRCas9 gene-editing of the PDCD1 locus in CAR T cells specific for EGFRvIII. CAR T cells are known to exist in various states of differentiation, with less differentiated stem cell memory $\left(\mathrm{T}_{\mathrm{SCM}}\right)$ or central memory $\left(\mathrm{T}_{\mathrm{CM}}\right)$ subtypes preferred over well-differentiated effector memory cells $\left(\mathrm{T}_{\mathrm{EM}}\right)$, specifically regarding characteristics such as expansion, persistence, and the capacity for self-renewal [10]. Moreover, loss of PD-1 has been shown to alter memory T-cell content and generation in other settings [11]. At baseline, both CART-EGFRvIII and CARTEGFRvIII $\triangle$ PD-1 demonstrated similar T-cell differentiation patterns compared to control $\mathrm{T}$ cells that had also been gene-edited for TRAC and B2M, in addition to undergoing mock transduction with AAV6 (Fig. 2, left column). By contrast, prolonged stimulation of CARTEGFRvIII $\triangle \mathrm{PD}-1$ led to a selective enrichment of $\mathrm{T}_{\mathrm{CM}}$, while CART-EGFRvIII cells expressing native PD-1 appeared to enrich for the more differentiated $\mathrm{T}_{\mathrm{EM}}$ compartment (Fig. 2, right column).

\section{PD-1 deletion promotes antitumor activity of CART- EGFRvIll in vitro}

Next, we turned our attention to the functional capacity of gene-edited CAR $\mathrm{T}$ cells in mediating antitumor immune responses in vitro. In experiments using primary human T cells, CART-EGFRvIII $\triangle \mathrm{PD}-1$ cells were found to produce significantly greater amounts of Th1 proinflammatory cytokines (e.g., IFN- $\gamma$ and TNF- $\alpha$ ) when cultured with EGFRvIII-expressing glioma compared to CAR T cells expressing endogenous PD-1 (Fig. 3a). We also compared each construct for the ability to initiate and maintain $\mathrm{T}$-cell proliferation. Following serial stimulation with irradiated target cells, repeated antigen stimulation through EGFRvIII maintained proliferation of both CART-EGFRvIII cells and CART-EGFRvIIISPD1 cells for over 1 month (Fig. 3b). Impedance-based, microelectronic platforms were then used to capture real-time kinetics of antitumor cytotoxicity as measured by target cell index (e.g., viability). Using this system, we found that CART-EGFRvIII $\triangle \mathrm{PD}-1$ cells were significantly more efficacious against U87vIII than those expressing PD-1, but that this difference was observed only after an extended period of time (Fig. 3c).

\section{CART-EGFRvIIILPD-1 cells are effective against EGFRvIII- expressing glioma}

Based on our observations in vitro, we proceeded to evaluate the function of CART-EGFRvIII $\triangle \mathrm{PD}-1$ in animal models of human glioma. First, we implanted tumors with stereotactic assistance into the brains of NSG (NOD.Cg-Prkdc ${ }^{\text {sci- }}$ ${ }^{\mathrm{d}} \mathrm{Il} 2 \mathrm{rg}^{\mathrm{tm} 1 \mathrm{Wjl}} /$ SzI) mice. This was followed by intravenous infusion of control, CART-EGFRvIII or CART-EGFRvIII $\triangle$ PD-1 cells via tail vein. Results did not demonstrate significantly prolonged survival in mice treated with EGFRvIII-specific CAR T cells systemically compared to the control (Fig. 4-c).

Because impressive results have been observed when administering CAR $\mathrm{T}$ cells intracranially-particularly into the ventricular system-in the setting of intracerebral tumors $[3,12,13]$, we reasoned that this might also represent the ideal route for delivery of CARTEGFRvIII $\triangle \mathrm{PD}-1$ cells. Indeed, following intraventricular infusion (Fig. 5a), treatment with CART-EGFRvIII $\triangle$ PD-1 cells led to significantly prolonged survival in mice with EGFRvIII-expressing glioma, including durable, complete cures in select mice (Fig. 5b,c). No long-term survivors developed clinical signs of xenogeneic GVHD.

\section{Discussion}

CARs have shown early potential in clinical trials for patients with GBM; however, treatment has been associated with marked upregulation of PD-L1 in glioma tissue, which can have profound, counterproductive effects on antitumor immunity [6]. Prior studies have demonstrated that CRISPR-Cas9 technology can be used to disrupt signaling through PD-1 in primary human $\mathrm{T}$ cells and to create potential "off-the-shelf," allogeneic CAR $\mathrm{T}$ cell products through simultaneous editing at the TRAC and $B 2 M$ loci [14-16]. In the current study, we have applied these approaches to generate universal, EGFRvIII-targeted CAR $\mathrm{T}$ cells resistant to PD-L1 checkpoint inhibition. In addition, we have demonstrated efficacy of these CAR $T$ cells in murine models of human GBM. Our findings also contribute to mounting data suggesting that route-of-administration may play a critical role in achieving optimal CAR T-cell activity against tumors in the brain. 


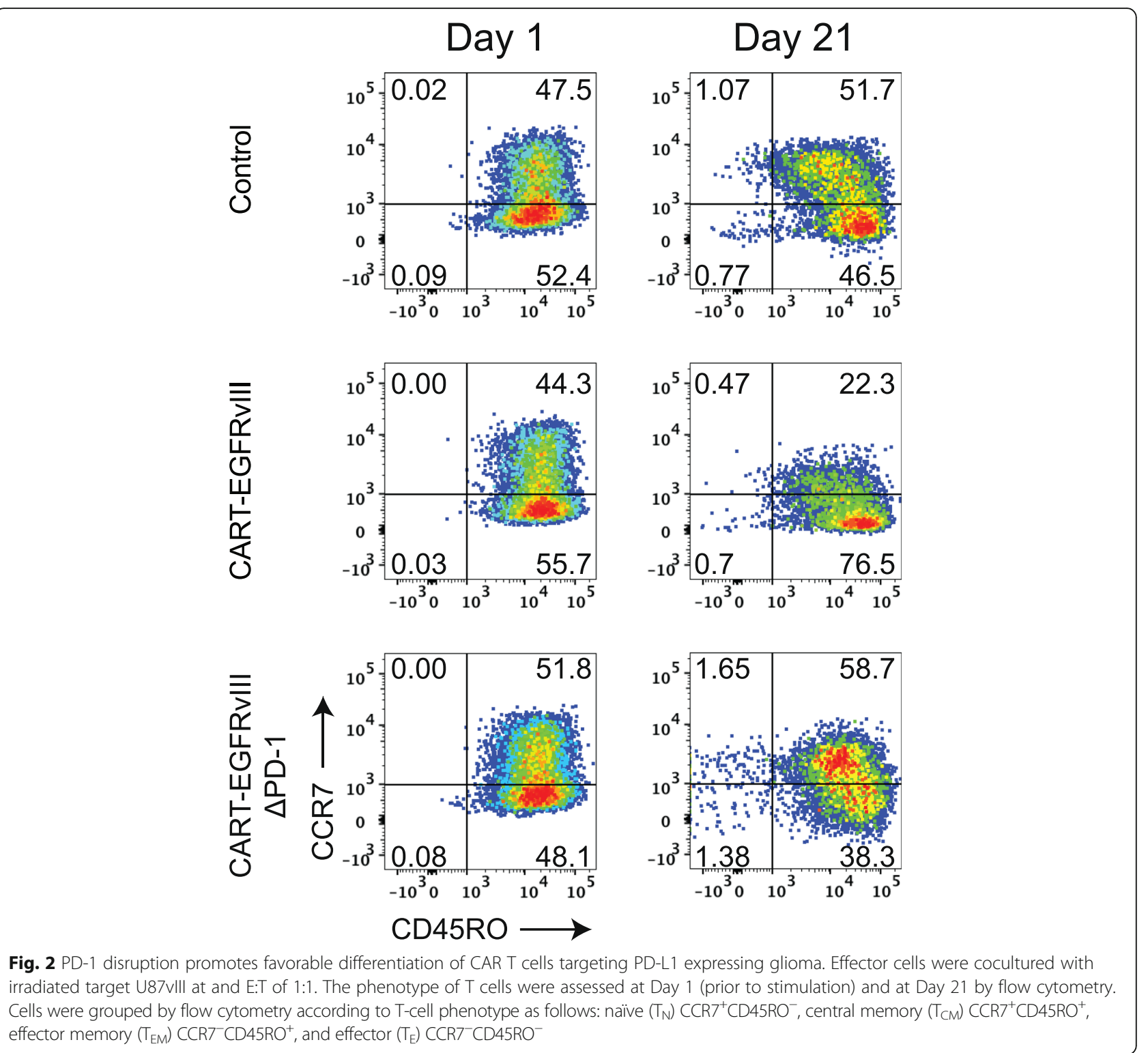

Recent work has highlighted immune checkpoint regulation through PD-1/PD-L1 as a promising therapeutic target in GBM. Aside from gene-editing techniques, a popular approach to targeting this pathway has been the use of immune checkpoint blockade (ICB) with monoclonal antibodies. Although ICB may potentially benefit certain subsets of patients with recurrent glioma [17-19], a randomized phase III study of PD-1/PD-L1 axis inhibition for GBM did not demonstrate prolonged overall survival [20]. Possible explanations for this have included concomitant chemotherapy-induced lymphopenia as well as structural considerations associated with the blood-brain barrier, which could impede interactions between systemically administered antibody and either infiltrating $\mathrm{T}$ cells or intracerebral tumor tissue. Unlike antibody therapies, CAR $\mathrm{T}$ cells have the ability to leverage profound lymphopenia to enhance antitumor activity following adoptive transfer into temozolomide-treated, lymphodepleted hosts [21, 22]. Engineering CAR T cells to secrete PD-1-blocking antibody fragments at targeted sites such as the tumor microenvironment has been proposed [23]. However, it has also been suggested that ICBs in these settings can act indiscriminately and may be responsible for hyper-progressive disease states due to unintended effects on suppressive $\mathrm{PD}-1^{+}$regulatory T-cell subsets [24]. In our study, we found that deletion of PD-1 in CAR T cells, where only CAR $\mathrm{T}$ cells have direct cytotoxic potential (i.e., there was no antigen spreading and no secondary immune activation), had minimal effects on efficacy. Together these data suggest that GBM will require additional technologies to enhance the therapeutic effects of T-cell immunity. 
a
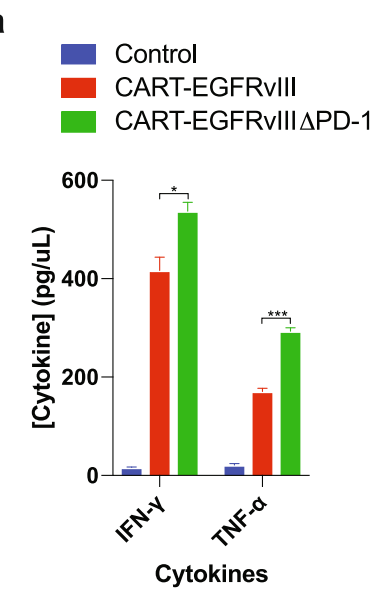

b

- Control

- CART-EGFRvIII

- CART-EGFRVIIIIPD-1

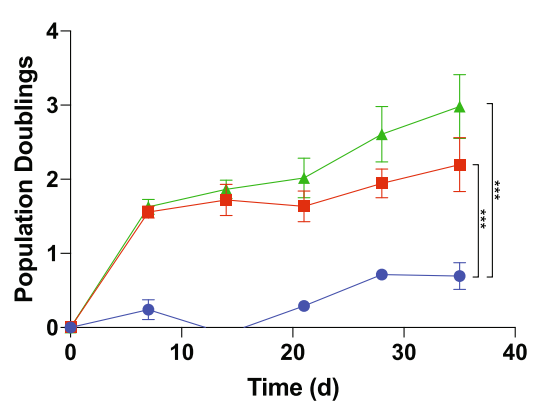

C

- Control

- CART-EGFRvIII

— CART-EGFRvIIISPD-1

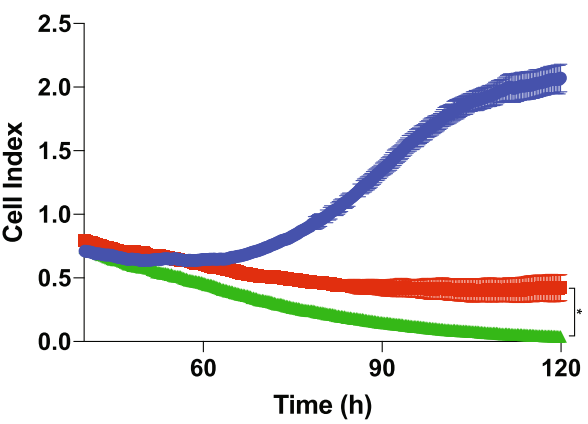

Fig. 3 PD-1 disruption enhances EGFRvIII CAR T cells. a Cytokine production by CAR-transduced primary human T cells when cocultured for $18 \mathrm{~h}$ at an E:T of 1:1. b Proliferation assessment of effector cells stimulated weekly with irradiated U87vIII. c Impedance-based cytotoxicity assay measuring activity of effector cells against U87vIll at an E:T of 1:3, with cell index serving as an inverse measure of target cell viability. Assays were performed in triplicate (mean \pm SEM is depicted; unpaired, two-tailed $t$-test, ${ }^{*}=P<0.05,{ }^{* * *}=p<0.001$ )

Locoregional immune therapy represents a particularly attractive route-of-delivery for tumors in the central nervous system (CNS), which are thought to be isolated to some degree from the peripheral circulation by a specialized blood-brain barrier. Indeed, several studies have supported that direct infusion of CAR $\mathrm{T}$ cells into the ventricular system of the brain may be necessary to achieve optimal antitumor activity, and in one case this approach was required to mediate the regression of bulky, multifocal, intracranial disease [3]. Benefits of intraventricular administration into cerebrospinal fluid (CSF) spaces include enhanced access to sites throughout the CNS as well as the ability to achieve adequate effector-to-target ratios, which represents a persistent challenge of cell therapy for solid tumors [5, 25].

In this study, we applied CRISPR-Cas9 as a tool to achieve multiplexed gene-editing of human CAR T cells.
Other methods of disrupting gene expression in $\mathrm{T}$ cells include the use of zinc finger nucleases (ZFN) [26] and TAL effector nucleases (TALEN) [27], though the use of these technologies has been relatively limited in targeting multiple genes simultaneously. Studies have shown that CRISPR can also be utilized to achieve concomitant gene integration and deletion. An example of this is a prior report of a CD19 CAR construct delivered directly into the TRAC locus, which also placed the transgene under control of an endogenous promoter [7]. Importantly, by virtue of these underlying mechanisms, CRISPR carries a certain risk of off-target mutagenesis. Several clinical studies are now open to evaluate the safety of this particular approach in primary human $\mathrm{T}$ cells; data from these trials have yet to be reported [28].

Currently, there exists a dearth of animal models that accurately recapitulate both intact immunity and antigen
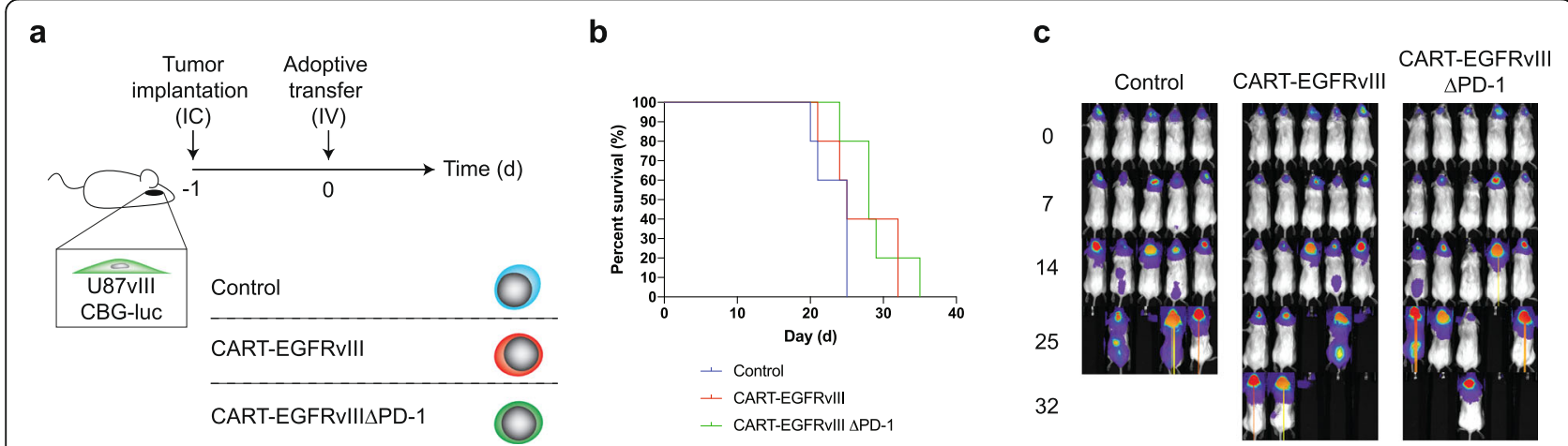

Fig. 4 Intravenous delivery of CAR T cells does not significantly prolong survival in mice. a U87vlll cells $\left(5 \times 10^{3}\right)$ were implanted orthotopically into NSG mice and treated post-implantation with intravenous (IV) effector cells. b Antitumor responses produced by CART-EGFRvIII $\triangle$ PD-1 in vivo. Survival curves were estimated for each group using Kaplan-Meier product-limit estimation. Primary comparative analyses of the curves for each group were performed using the log-rank test. c Bioluminescence imaging of U87vill tumor growth over time, $n=5$ mice 

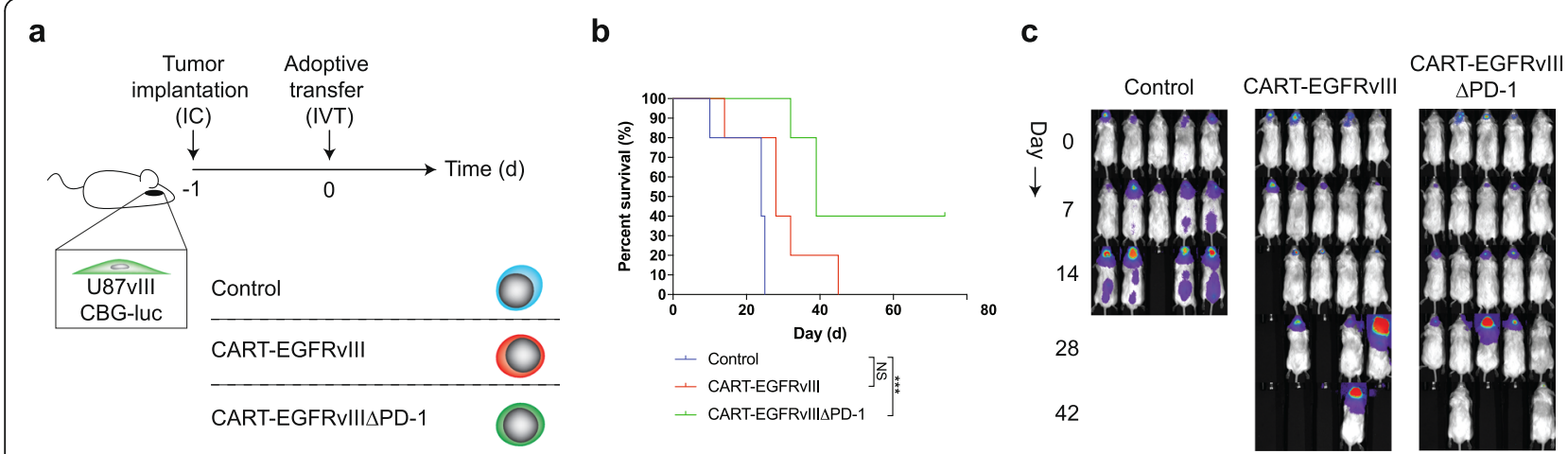

Fig. 5 Intraventricular infusion with gene-edited CAR T cells is efficacious against GBM. a U87vlll cells $\left(5 \times 10^{3}\right)$ were implanted orthotopically into NSG mice and treated post-implantation with intraventricular (IVT) effector cells. $\mathbf{b}$ Antitumor responses produced by CART-EGFRvIIIIPD-1 in vivo. Survival curves were estimated for each group using Kaplan-Meier product-limit estimation. Primary comparative analyses of the curves for each group were performed using the log-rank test $\left({ }^{* * *}=p<0.001\right)$. c Bioluminescence imaging of U87vlll tumor growth over time, $n=5$ mice

expression that would be encountered in a clinical setting. We elected the NSG mouse model to test our EGFRvIII CAR $\mathrm{T}$ cells as it permitted evaluation of a translatable human cell therapy along with the use of a human glioma cell line. One disadvantage of this approach is that it is unsuitable for experiments that seek to directly determine the efficacy of TCR and B2M deletion on GVHD or donor T-cell rejection, respectively. Ultimately, clinical trials may be the only appropriate way to definitely assess safety of these cell products in humans.

To our knowledge, this is the first report of triple deletion of TRAC, B2M and PDCD1 in CAR T cells tested in a solid tumor model. The results obtained with CARTEGFRvIII $\triangle$ PD-1 directly address shortcomings we noted during our clinical trial of EGFRvIII-targeted CARs and thus warrant further investigation in patients with GBM.

\section{Methods}

\section{Study design}

In this study, we sought to apply CRISPR-Cas9 technology to EGFRvIII CAR T cells in order to address extant barriers to achieving maximal therapeutic efficacy for patients with GBM. Specifically, we created EGFRvIII-specific CAR $\mathrm{T}$ cells with targeted deletion of PD-1 in order to make them resistant to immune checkpoint signaling through this pathway. In addition, we used this approach to simultaneously disrupt loci corresponding to genes for both endogenous T-cell receptor (TCR) and beta-2 microglobulin (B2M). We used several preclinical modeling systems to test our hypotheses, including in vitro and in vivo platforms. These consisted of phenotypic and functional assays. Direct antitumor activity was tested against human glioma cell line targets transduced to express EGFRvIII. In this manuscript, $\mathrm{T}$ cells from a single healthy donor batch preparation were used, as would be used in a trial setting. Cells were not purified after genetic manipulation. The
CAR T cells used in vitro were isolated from the same Tcell expansion as those used in vivo. Experiments were performed multiple times with representative data shown.

\section{Mice and cell lines}

Immune compromised NSG mice were originally purchased from Jackson Laboratory and bred under pathogen-free conditions, according to protocols approved by the Institutional Animal Care and Use Committee. Human glioma cell lines U87 and U251 were obtained from American Type Culture Collection (ATCC) and cultured under conditions as outlined by the supplier. The U87vIII cell line was generated by lentiviral transduction.

\section{CAR T-cell production}

CAR $T$ cell constructs were synthesized and cloned into an AAV6 plasmid backbone. All constructs included a CD8 transmembrane domain in tandem with an intracellular 4-1BB costimulatory and $\mathrm{CD} 3 \zeta$ signaling domain. Gene-editing and cell preparation was performed using standard techniques as described in detail elsewhere [29]. Briefly, human peripheral blood mononuclear cells (PBMCs) were thawed and the $\mathrm{T}$ cells were activated with conjugated CD3/CD28 agonists for 3 days in T-cell media containing human serum, IL-2 and IL-7. After activation, the $\mathrm{T}$ cells were electroporated with Cas9 protein and sgRNAs targeting the TRAC and $B 2 M$ loci or TRAC, $B 2 M$, and $P D C D 1$ loci and subsequently transduced with a recombinant AAV6 vector containing donor template DNA for insertion of the EGFRvIII CAR construct, with a typical transduction efficiency of $35 \%$. Following electroporation and transduction, the CAR T cells were expanded for 7 days in T-cell media containing human serum, IL-2, and IL-7. These cells were subsequently transferred to storage in liquid nitrogen prior to assays. 


\section{T-cell assays}

T-cell assays for activity, proliferation and cytotoxicity have been described in detail elsewhere [30]. Briefly, in coculture experiments, $T$ cells were incubated with irradiated U87vIII target cells at an E:T of 1:1 for time periods as described. Cell-free supernatants from cells were also analyzed for cytokine expression using a Luminex array (Luminex Corp, FLEXMAP 3D) according to manufacturers instructions. Expression of surface markers were either taken at baseline or after a period of coculture, and then subjected to flow cytometric analysis. Antigens were stained for using the following antibody clones for flow cytometry where indicated: CCR7 (3E12, BD Bioscience); CD45RO (UCHL1, BD Biosciences), PD-1 (EH12287, Biolegend). For proliferation assays, cells were stimulated with irradiated target cells at an E:T of 1:1. Cells were counted every 7 days and plated again with stimulation at 7 day intervals. In experiments when real-time cytotoxicity was measured against U87vIII, cell index was recorded as a measure of cell impedance using the xCELLigence RTCA SP instrument (ACEA Biosciences, Inc.) according to manufacturer instructions. Percent specific lysis may be calculated from these data using the following equation: $\%=(($ cell index of UTDs - cell index of CAR T cells $) /$ cell index of UTDs) $\times 100$.

\section{Animal models}

Tumor cells were harvested in logarithmic growth phase, counted, and loaded in a $50 \mu \mathrm{L}$ syringe with an attached 25 -gauge needle. Mice were anesthetized and placed in a stereotactic frame to assist in tumor implantation. Tumor cells were implanted at $2 \mathrm{~mm}$ to the right of the bregma at a depth of $4 \mathrm{~mm}$ from the surface of the skull, in a total volume of $5 \mu \mathrm{L}$. Effector cells were then infused systemically by tail vein infusion in a total volume of $100 \mu \mathrm{L}$ or administered intraventricularly in a total volume of $30 \mu \mathrm{L}$. Intraventricular delivery was at $2 \mathrm{~mm}$ to the left of and $0.3 \mathrm{~mm}$ anterior to the bregma at a depth of $3 \mathrm{~mm}$ from the surface of the skull. Effector cell populations were normalized to contain $1 \times 10^{6}$ cells per infusion for all experiments. Tumor progression was assessed over time by bioluminescence emission using the Ami HT optical imaging system (Spectral Instruments) following intraperitoneal luciferin injection. Survival was determined by mice found expired or otherwise sacrificed by a blinded technician at predetermined humane endpoints.

\section{Statistical methods}

All analyses were performed with GraphPad Prism 7.0c software. Data was presented as means \pm SEM with statistically significant differences determined by tests as indicated in figure legends.

\section{Supplementary information}

Supplementary information accompanies this paper at https://doi.org/10. 1186/s40425-019-0806-7.

Additional file 1: Figure S1. PD-L1 expression on glioblastoma cell lines. Flow cytometric analysis of PD-L1 on human glioma cell lines versus isotype control.

\section{Abbreviations}

B2M : beta-2 microglobulin; CAR: chimeric antigen receptor; CNS: central nervous system; CSF: cerebrospinal fluid; GBM: glioblastoma; GVHD: graftversus-host disease; PD-L1: programmed cell death ligand 1; TALEN: TAL effector nuclease; TCR: T-cell receptor; TRAC: T-cell receptor alpha constant; ZFN: zinc finger nuclease

\section{Acknowledgements}

We thank Lauren Riley and Maria Cabral-Rodriguez for their expert technical assistance.

\section{Authors' contributions}

$B D C, X Y$, and MVM designed research; BDC, XY, APC, HD, DH, AAB, and GMG performed experiments; BDC, WTC, BSC, and MVM contributed reagents and analytic tools; BDC, XY, APC, AAB, RCL, IS, SRB, MJF, AS, and MBL analyzed and interpreted data; $B D C, X Y, J S$ and MVM designed research and wrote the paper. The authors are solely responsible for the content of the research and data presented herein. All authors read and approved the final manuscript.

\section{Funding}

This work was partially supported by a sponsored research agreement with CRISPR Therapeutics. This work was supported by grants from the National Institutes of Health R25NS065743 (B.D.C.), the Neurosurgery Research \& Education Foundation and B*Cured Research Fellowship Grant (B.D.C), and the Society for Immunotherapy of Cancer-AstraZeneca Postdoctoral Cancer Immunotherapy in Combination Therapies Clinical Fellowship Award (B.D.C). This work was also supported by the Damon Runyon-Rachleff Innovation Award (M.V.M) and Stand Up to Cancer (M.V.M). We thank the following core facilities of the MGH Cancer Center: MGH Blood Bank, Flow Cytometry, Confocal Microscopy, and Histopathology.

\section{Availability of data and materials}

Data generated in this study are available from the corresponding author.

\section{Ethics approval and consent to participate}

All experiments in this study were performed under protocols reviewed by the Massachusetts General Hospital Institutional Animal Care and Use Committee.

\section{Consent for publication}

Not applicable.

\section{Competing interests}

B.D.C. and M.V.M are inventors on patents related to the use of engineered cell therapies and bispecific T-cell engagers for GBM and other cancers. B.D.C. received commercial research grants from ACEA Biosciences. M.V.M. received commercial research grants from Kite Pharma, TCR2, Agentus, and CRISPR Therapeutics, and is a consultant or advisory board member for Adaptimmune, Agentus, Cellectis, CRISPR Therapeutics, Kite Pharma, Novartis, TCR2, and Windmil (unrelated to this work). H.D., D.B.H., and J.G.S. are employees at CRISPR Therapeutics who possess stock or options in the company.

\section{Author details}

${ }^{1}$ Cellular Immunotherapy Program, Cancer Center, Massachusetts General Hospital and Harvard Medical School, 149 13th Street, Room 3.216, Charlestown, Boston, Massachusetts 02129, USA. ²Department of Neurosurgery, Massachusetts General Hospital and Harvard Medical School, Boston, Massachusetts, USA. ${ }^{3}$ CRISPR Therapeutics, Cambridge, Massachusetts, USA. ${ }^{4}$ Department of Medicine, Massachusetts General Hospital and Harvard Medical School, Boston, Massachusetts, USA. 
Received: 29 May 2019 Accepted: 6 November 2019

Published online: 14 November 2019

\section{References}

1. Louis DN, Perry A, Reifenberger G, von Deimling A, Figarella-Branger D, Cavenee WK, et al. The 2016 World Health Organization classification of tumors of the central nervous system: a summary. Acta Neuropathol. 2016; 131(6):803-20.

2. Stupp R, Hegi ME, Mason WP, van den Bent MJ, Taphoorn MJ, Janzer RC, et al. Effects of radiotherapy with concomitant and adjuvant temozolomide versus radiotherapy alone on survival in glioblastoma in a randomised phase III study: 5-year analysis of the EORTC-NCIC trial. Lancet Oncol. 2009; 10(5):459-66.

3. Brown CE, Alizadeh D, Starr R, Weng L, Wagner JR, Naranjo A, et al. Regression of Glioblastoma after chimeric antigen receptor T-cell therapy. N Engl J Med. 2016;375(26):2561-9.

4. Mullard A. FDA approves first CAR T therapy. Nat Rev Drug Discov. 2017; 16(10):669.

5. Choi BD, Maus MV, June CH, Sampson JH. Immunotherapy for Glioblastoma: adoptive T-cell strategies. Clin Cancer Res. 2019;25(7):2042-8.

6. O'Rourke DM, Nasrallah MP, Desai A, Melenhorst JJ, Mansfield K, Morrissette JJD, et al. A single dose of peripherally infused EGFRvIll-directed CAR T cells mediates antigen loss and induces adaptive resistance in patients with recurrent glioblastoma. Sci Transl Med. 2017:9:399.

7. Eyquem J, Mansilla-Soto J, Giavridis T, van der Stegen SJ, Hamieh M, Cunanan KM, et al. Targeting a CAR to the TRAC locus with CRISPR/Cas9 enhances tumour rejection. Nature. 2017;543(7643):113-7.

8. Torikai H, Reik A, Soldner F, Warren EH, Yuen C, Zhou Y, et al. Toward eliminating HLA class I expression to generate universal cells from allogeneic donors. Blood. 2013;122(8):1341-9.

9. Nduom EK, Wei J, Yaghi NK, Huang N, Kong LY, Gabrusiewicz K, et al. PD-L1 expression and prognostic impact in glioblastoma. Neuro-Oncology. 2016; 18(2):195-205.

10. Sadelain M, Riviere I, Riddell S. Therapeutic T cell engineering. Nature. 2017; 545(7655):423-31.

11. Allie SR, Zhang W, Fuse S, Usherwood EJ. Programmed death 1 regulates development of central memory CD8 T cells after acute viral infection. J Immunol. 2011;186(11):6280-6.

12. Priceman SJ, Tilakawardane D, Jeang B, Aguilar B, Murad JP, Park AK, et al. Regional delivery of chimeric antigen receptor-engineered $T$ cells effectively targets HER2(+) breast Cancer metastasis to the brain. Clin Cancer Res. 2018; 24(1):95-105.

13. Choi BD, Suryadevara CM, Gedeon PC, Herndon JE 2nd, Sanchez-Perez L, Bigner DD, et al. Intracerebral delivery of a third generation EGFRvIII-specific chimeric antigen receptor is efficacious against human glioma. J Clin Neurosci. 2014;21(1):189-90.

14. Su S, Hu B, Shao J, Shen B, Du J, Du Y, et al. CRISPR-Cas9 mediated efficient PD-1 disruption on human primary T cells from cancer patients. Sci Rep. 2016;6:20070

15. Su S, Zou Z, Chen F, Ding N, Du J, Shao J, et al. CRISPR-Cas9-mediated disruption of PD-1 on human T cells for adoptive cellular therapies of EBV positive gastric cancer. Oncoimmunology. 2017;6(1):e1249558.

16. Ren J, Liu X, Fang C, Jiang S, June CH, Zhao Y. Multiplex genome editing to generate universal CAR T cells resistant to PD1 inhibition. Clin Cancer Res. 2017;23(9):2255-66.

17. Cloughesy TF, Mochizuki AY, Orpilla JR, Hugo W, Lee AH, Davidson TB, et al. Neoadjuvant anti-PD-1 immunotherapy promotes a survival benefit with intratumoral and systemic immune responses in recurrent glioblastoma. Nat Med. 2019:25(3):477-86.

18. Schalper KA, Rodriguez-Ruiz ME, Diez-Valle R, Lopez-Janeiro A, Porciuncula A, Idoate MA, et al. Neoadjuvant nivolumab modifies the tumor immune microenvironment in resectable glioblastoma. Nat Med. 2019;25(3):470-6.

19. Zhao J, Chen AX, Gartrell RD, Silverman AM, Aparicio L, Chu T, et al. Immune and genomic correlates of response to anti-PD-1 immunotherapy in glioblastoma. Nat Med. 2019;25(3):462-9.

20. Reardon DA, Omuro A, Brandes AA, Rieger J, Wick A, Sepulveda J, et al. OS10.3 Randomized Phase 3 Study Evaluating the Efficacy and Safety of Nivolumab vs Bevacizumab in Patients With Recurrent Glioblastoma: CheckMate 143. Neuro-Oncology. 2017;19(Suppl 3):iii21-ii.

21. Sanchez-Perez LA, Choi BD, Archer GE, Cui X, Flores C, Johnson LA, et al. Myeloablative temozolomide enhances CD8(+) T-cell responses to vaccine and is required for efficacy against brain tumors in mice. PLoS One. 2013; 8(3):e59082.

22. Suryadevara CM, Desai R, Abel ML, Riccione KA, Batich KA, Shen SH, et al. Temozolomide lymphodepletion enhances CAR abundance and correlates with antitumor efficacy against established glioblastoma. Oncoimmunology. 2018;7(6):e1434464.

23. Rafiq S, Yeku OO, Jackson HJ, Purdon TJ, van Leeuwen DG, Drakes DJ, et al. Targeted delivery of a PD-1-blocking scFv by CAR-T cells enhances antitumor efficacy in vivo. Nat Biotechnol. 2018;36(9):847-56.

24. Kamada T, Togashi Y, Tay C, Ha D, Sasaki A, Nakamura Y, et al. PD-1(+) regulatory T cells amplified by PD-1 blockade promote hyperprogression of cancer. Proc Natl Acad Sci U S A. 2019.

25. Choi BD, Curry WT, Carter BS, Maus MV. Chimeric antigen receptor T-cell immunotherapy for glioblastoma: practical insights for neurosurgeons. Neurosurg Focus. 2018:44(6):E13.

26. Provasi E, Genovese P, Lombardo A, Magnani Z, Liu PQ, Reik A, et al. Editing $T$ cell specificity towards leukemia by zinc finger nucleases and lentiviral gene transfer. Nat Med. 2012;18(5):807-15.

27. Berdien B, Mock U, Atanackovic D, Fehse B. TALEN-mediated editing of endogenous $T$-cell receptors facilitates efficient reprogramming of $\mathrm{T}$ lymphocytes by lentiviral gene transfer. Gene Ther. 2014;21 (6):539-48.

28. Singh N, Shi J, June CH, Ruella M. Genome-editing Technologies in Adoptive T Cell Immunotherapy for Cancer. Curr Hematol Malig Rep. 2017; 12(6):522-9.

29. Albers JJ, Ammon T, Gosmann D, Audehm S, Thoene S, Winter C, et al. Gene editing enables T-cell engineering to redirect antigen specificity for potent tumor rejection. Life Sci Alliance. 2019;2(2).

30. Choi BD, Yu X, Castano AP, Bouffard AA, Schmidts A, Larson RC, et al. CAR-T cells secreting BiTEs circumvent antigen escape without detectable toxicity. Nat Biotechnol. 2019;37(9):1049-58.

\section{Publisher's Note}

Springer Nature remains neutral with regard to jurisdictional claims in published maps and institutional affiliations.

Ready to submit your research? Choose BMC and benefit from:

- fast, convenient online submission

- thorough peer review by experienced researchers in your field

- rapid publication on acceptance

- support for research data, including large and complex data types

- gold Open Access which fosters wider collaboration and increased citations

- maximum visibility for your research: over $100 \mathrm{M}$ website views per year

At $\mathrm{BMC}$, research is always in progress.

Learn more biomedcentral.com/submissions 